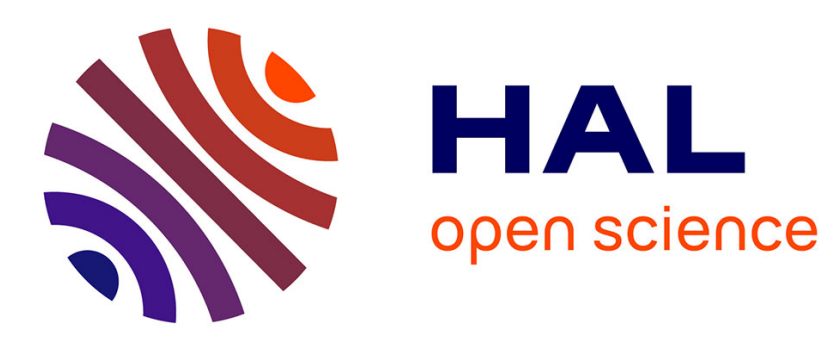

\title{
Target detection in active polarization images perturbed with additive noise and illumination nonuniformity.
}

Arnaud Bénière, François Goudail, Daniel Dolfi, Mehdi Alouini

\section{To cite this version:}

Arnaud Bénière, François Goudail, Daniel Dolfi, Mehdi Alouini. Target detection in active polarization images perturbed with additive noise and illumination nonuniformity.. Journal of the Optical Society of America. A Optics, Image Science, and Vision, 2009, 26 (7), pp.1678-86. 10.1364/JOSAA.26.001678 . hal-00665140

\section{HAL Id: hal-00665140 \\ https://hal.science/hal-00665140}

Submitted on 6 Apr 2012

HAL is a multi-disciplinary open access archive for the deposit and dissemination of scientific research documents, whether they are published or not. The documents may come from teaching and research institutions in France or abroad, or from public or private research centers.
L'archive ouverte pluridisciplinaire HAL, est destinée au dépôt et à la diffusion de documents scientifiques de niveau recherche, publiés ou non, émanant des établissements d'enseignement et de recherche français ou étrangers, des laboratoires publics ou privés. 


\title{
Target detection in active polarization images perturbed with additive noise and illumination nonuniformity
}

\author{
Arnaud Bénière, ${ }^{1,2}$ François Goudail, ${ }^{1, *}$ Daniel Dolfi, ${ }^{2}$ and Mehdi Alouini ${ }^{2,3}$ \\ ${ }^{1}$ Laboratoire Charles Fabry de l'Institut d'Optique, CNRS, Univ Paris-Sud, Campus Polytechnique, RD 128, \\ 91127 Palaiseau, France \\ ${ }^{2}$ Thales Research and Technology-France, RD128, 91767 Palaiseau Cedex, France \\ ${ }^{3}$ Institut de Physique de Rennes, CNRS, Université de Rennes 1, 35042 Rennes, France \\ *Corresponding author: francois.goudail@institutoptique.fr
}

Received January 14, 2009; accepted April 25, 2009; posted May 27, 2009 (Doc. ID 106386); published June 24, 2009

\begin{abstract}
Active imaging systems that illuminate a scene with polarized light and acquire two images in two orthogonal polarizations yield information about the intensity contrast and the orthogonal state contrast (OSC) in the scene. Both contrasts are relevant for target detection. However, in real systems, the illumination is often spatially or temporally nonuniform. This creates artificial intensity contrasts that can lead to false alarms. We derive generalized likelihood ratio test (GLRT) detectors, for which intensity information is taken into account or not and determine the relevant expressions of the contrast in these two situations. These results are used to determine in which cases considering intensity information in addition to polarimetric information is relevant or not. (C) 2009 Optical Society of America

OCIS codes: 260.543, 030.4280 .
\end{abstract}

\section{INTRODUCTION}

Imaging systems that measure the degree of polarization (DOP) of light backscattered by a scene have aroused great interest in several domains such as machine vision [1], biomedical imaging [2,3], and remote sensing [4,5]. These systems can, for example, reveal contrasts between regions of a scene which have the same intensity reflectivity but different polarimetric properties [6]. One can, for instance, distinguish a metallic object from one made with plastic even if they have the same reflectance spectrum, and improve the visibility through diffusive (fog, liquids,...) [7] or turbulent media (atmosphere). A simple configuration consists of illuminating the scene with a totally polarized beam (whose state of polarization is located anywhere on the Poincaré sphere) and acquiring two images: the first one, $X$, is formed with the fraction of the backscattered light having the same state of polarization as the illumination and the second one, $Y$, is formed with the fraction of the light in the orthogonal state [8-10]. From these two images, the standard intensity image $X+Y$ and the orthogonal state contrast (OSC) image [11] $(X-Y) /(X+Y)$ can be computed.

The OSC image represents the DOP of the backscattered light if the observed materials are purely depolarizing [6]. Moreover, it is insensitive to variations of illumination intensity. This is an important advantage since in real active imaging systems, the illumination pattern may be nonuniform owing to spatial or temporal fluctuations of the light source. This creates artificial intensity contrasts that can lead to false alarms. One way to avoid this problem is to base the detection on the OSC only, that is, not to take into account intensity information. One is no longer affected by intensity fluctuations of the source, but if these fluctuations are weak and reflectance contrast is actually present in the scene, discarding intensity information may lead to a decrease of detection performance.

The first objective of this paper is to derive generalized likelihood ratio test (GLRT) detectors in two situations: intensity information is assumed irrelevant or not. We shall deduce from their expressions the adequate definition of the contrast in these two situations. These results will be used to determine in which cases taking intensity information into account is relevant or not.

The paper is organized as follows. In Section 2, we define the data model and describe the formalism of our detection problem. Section 3 is devoted to the derivation of the GLRT when the illumination is nonuniform but known. The case of uniform illumination is treated as a special case. In Section 4, the GLRT is calculated when the illumination is unknown. We finally compare in Section 5 the performances of these detectors based on their receiver operating characteristic (ROC) and on simulated images.

\section{DESCRIPTION OF THE PROBLEM}

We consider subsamples $X_{i}$ and $Y_{i}, i \in[1, N]$, of the images, that may be spatial if we consider a small set of neighboring pixels, or temporal if we consider a single pixel in several successive acquisitions. To permit a rigorous treatment of estimation and detection properties, each sample is assumed homogeneous, that is, the average number of photoelectrons under unitary and uniform 
illumination, denoted $m_{X}$ and $m_{Y}$, is identical for all pixels. The intensity for the two polarization directions at each pixel $i \in[1, N]$ can be written as

$$
\begin{aligned}
& X_{i}=F_{i} m_{X}+n_{i}^{x}, \\
& Y_{i}=F_{i} m_{Y}+n_{i}^{y},
\end{aligned}
$$

where $\mathbf{F}$ denotes the spatial fluctuations of the illumination intensity. In other words, $F_{i}$ represents the intensity of the incoming light and $m_{X}\left(m_{Y}\right)$ represent the reflectivity of the observed material with respect to each polarization. Each measure is perturbed by additive white Gaussian noise $n_{i}^{x}$ and $n_{i}^{y}$ with zero mean and variance $\sigma^{2}$, which are assumed statistically independent.

The problem of OSC estimation has been widely addressed with different types of noise, such as for example speckle noise, coupled photon and speckle noise, and detector noise [12-14]. More recently, we addressed the case of additive and signal-dependent photon noise [15], which presents great similarities with the case of additive Gaussian noise [16]. Concerning detection and segmentation, the case of speckle noise has been studied, for example, in $[17,18]$. In this paper, we will assume that the active illumination is weakly coherent so that speckle noise is neglected and additive detector noise is dominant. This is a realistic case from a system point of view.

The two parameters of interest in the sample are the intensity reflectivity of the material $I=m_{X}+m_{Y}$ and the OSC parameter defined as

$$
P=\frac{m_{X}-m_{Y}}{m_{X}+m_{Y}} .
$$

The OSC represents the DOP of the backscattered light only if the observed materials are purely depolarizing. This is a reasonable assumption for natural materials and turbid media observed in monostatic (backscattering) configuration with linearly polarized illumination [6]. In the general case, $P$ can still provide a source of contrast useful for detection.

The data form a sample $\chi=\{\mathbf{X}, \mathbf{Y}\}$ where $\mathbf{X}=\left\{X_{i}, i\right.$ $\in[1, N]\}$ and $\mathbf{Y}=\left\{Y_{i}, i \in[1, N]\right\}$. Its statistical properties are defined by the noise variance $\sigma^{2}$, which is assumed known, and by the parameters of interest $\left(m_{X}, m_{Y}\right)$ or, what is equivalent, $(I, P)$. Actually one has

$$
m_{X}=I \frac{1+P}{2}, \quad m_{Y}=I \frac{1-P}{2} .
$$

To address detection, we will use the formalism explained in detail in [19]. We will consider that the sample is divided into two parts of size $N_{a}$ and $N_{b}$ so that $N_{a}$ $+N_{b}=N$. In order to simplify the notation we define $\Omega_{a}$ $=\left[1, N_{a}\right]$ and $\Omega_{b}=\left[N_{a}+1, N\right]$. The first part of the subsample $\chi_{a}=\left\{X_{i}, Y_{i}, i \in \Omega_{a}\right\}$ is defined by parameters $\left(m_{X}^{a}, m_{Y}^{a}\right)$ or $\left(I_{a}, P_{a}\right)$. The second part of the subsample $\chi_{b}$ $=\left\{X_{i}, Y_{i}, i \in \Omega_{b}\right\}$ is defined by the parameters $\left(m_{X}^{b}, m_{Y}^{b}\right)$ or $\left(I_{b}, P_{b}\right)$. We denote $\mathbf{F}_{\mathbf{a}}=\left[F_{i}\right]_{i \in \Omega_{a}}$ and $\mathbf{F}_{\mathbf{b}}=\left[F_{i}\right]_{i \in \Omega_{b}}$. We will consider the following hypothesis testing problem:

- Hypothesis $H_{1}$ : the samples $\chi_{a}$ and $\chi_{b}$ have different parameters: $\left(I_{a}, P_{a}\right) \neq\left(I_{b}, P_{b}\right)$,
- Hypothesis $H_{0}$ : the samples $\chi_{a}$ and $\chi_{b}$ have the same parameters: $\left(I_{a}, P_{a}\right)=\left(I_{b}, P_{b}\right)$.

This model can account for the detection of an edge or of a target with homogeneous texture on a background. We will first consider that the illumination is known and determine the expression of GLRT in order to set a benchmark for detection performance. Then, we will consider the more realistic case of unknown illumination pattern.

\section{GLRT AND CONTRAST IN THE CASE WHERE ILLUMINATION PATTERN F IS KNOWN}

In this section, we will consider that the illumination pattern $\mathbf{F}$ is known, but that $m_{X}$ and $m_{Y}$, which are the target and background characteristics, are unknown.

Determining the GLRT [20] requires the determination of the profile log-likelihood of a homogeneous region, which is denoted $\ell(\chi \mid \mathbf{F})$. From this expression, we derive the GLRT $\mathcal{R}_{\text {Fknown }}=\ell_{1}(\chi \mid \mathbf{F})-\ell_{0}(\chi \mid \mathbf{F})$, where $\ell_{1}(\chi \mid \mathbf{F})$ is the profile log-likelihood in hypothesis $H_{1}$ and $\ell_{0}(\chi \mid \mathbf{F})$ the profile log-likelihood in hypothesis $H_{0}$. After some computations detailed in Appendix A, we obtain

$$
\mathcal{R}_{\text {Fknown }}=\frac{1}{2 \sigma^{2}} \frac{\Phi_{a} \Phi_{b}}{\Phi_{a}+\Phi_{b}}\left[\left(\hat{m}_{X}^{a}-\hat{m}_{X}^{b}\right)^{2}+\left(\hat{m}_{Y}^{a}-\hat{m}_{Y}^{b}\right)^{2}\right],
$$

where

$$
\Phi_{v}=\sum_{i \in \Omega_{v}} F_{i}^{2}, \quad \hat{m}_{U}^{v}=\frac{1}{\Phi_{v}} \sum_{i \in \Omega_{v}} F_{i} U_{i},
$$

with $U=\{X, Y\}$ and $v=\{a, b\}$.

The special case of uniform illumination corresponds to $F_{i}=F_{0}, \forall i \in[1, N]$. The expression of the GLRT in this case is easily derived from Eq. (4) and one gets

$$
\begin{aligned}
\mathcal{R}_{\text {uni }}= & \frac{1}{2 \sigma^{2}} \frac{N_{a} N_{b}}{N_{a}+N_{b}}\left[\left(\sum_{i \in \Omega_{a}} \frac{X_{i}}{N_{a}}-\sum_{i \in \Omega_{b}} \frac{X_{i}}{N_{b}}\right)^{2}\right. \\
& \left.+\left(\sum_{i \in \Omega_{a}} \frac{Y_{i}}{N_{a}}-\sum_{i \in \Omega_{b}} \frac{Y_{i}}{N_{b}}\right)^{2}\right] .
\end{aligned}
$$

In this case the GLRT depends only on the measurement data $\mathbf{X}$ and $\mathbf{Y}$.

Let us denote $\mathcal{R}_{\text {Fknown }}^{0}\left(\mathcal{R}_{\text {Fknown }}^{1}\right)$ the expression of the GLRT $\mathcal{R}_{\text {Fknown }}$ in hypothesis $H_{0}\left(H_{1}\right)$. The detection performance of the GLRT depends on the probability density functions (PDF) of these two random variables. We show in Appendix B that $2 \mathcal{R}_{\text {Fknown }}^{0}$ is a chi-square random variable with two degrees of freedom [21]. It is thus independent of the signal parameters $I$ and $P$, which makes $\mathcal{R}_{\text {Fknown }}$ a constant false alarm rate (CFAR) detector. We also show in Appendix B that $2 \mathcal{R}_{\text {Fknown }}^{1}$ is a noncentral chi-square variable with two degrees of freedom and noncentrality parameter equal to

$$
\begin{aligned}
\mathcal{C}_{\text {Fknown }}= & \frac{\Phi_{a} \Phi_{b}}{\left(\Phi_{a}+\Phi_{b}\right)}\left[\left(\mathrm{SNR}_{a}-\mathrm{SNR}_{b}\right)^{2}\right. \\
& \left.+\left(\mathrm{SNR}_{a} P_{a}-\mathrm{SNR}_{b} P_{b}\right)^{2}\right]
\end{aligned}
$$

with $\mathrm{SNR}_{a}=I_{a} /(\sqrt{2} \sigma)$ and $\mathrm{SNR}_{b}=I_{b} /(\sqrt{2} \sigma)$. It is seen that 
$\mathcal{R}_{\text {Fknown }}^{1}$, and thus the detection performance, depends only on the parameter $\mathcal{C}_{\text {Fknown }}$, which can thus be considered as the adequate contrast parameter for the detection problem at hand. In other words, if we consider two scenarios with identical contrast parameters $\mathcal{C}_{\text {Fknown }}$, the performances of the detector $\mathcal{R}_{\text {Fknown }}$ will be identical.

A particularly interesting case occurs when there is no intensity contrast between the regions $a$ and $b$, that is, $I_{a}=I_{b}=I$, or, in other words, $\mathrm{SNR}_{a}=\mathrm{SNR}_{b}=\mathrm{SNR}$. In this case, one has

$$
\mathcal{C}_{\text {Fknown }}=\frac{\Phi_{a} \Phi_{b}}{\left(\Phi_{a}+\Phi_{b}\right)} \operatorname{SNR}^{2}\left(P_{a}-P_{b}\right)^{2} .
$$

In this situation, the contrast $\mathcal{C}_{\text {Fknown }}$ depends on the difference of the DOP in the two regions weighted by the common value of the intensity SNR.

\section{GLRT IN THE CASE WHERE THE ILLUMINATION PATTERN F IS UNKNOWN}

The algorithm described above, which assumes that the illumination pattern $\mathbf{F}$ is known, can be used if this pattern is static and has been estimated beforehand. However, in many practical situations this is not the case, and $\mathbf{F}$ must be considered unknown. In this case, $\mathbf{F}$ constitutes a vectorial nuisance parameter. As in Section 3 we determine the profile log-likelihood of a homogeneous region, which is a simple but cumbersome computation detailed in Appendix C. This result is then used in Appendix $\mathrm{D}$ to obtain the following expression of the GLRT:

$$
\begin{aligned}
\mathcal{R}_{\text {Funknown }}= & \frac{1}{4 \sigma^{2}}\left\{\sqrt{D_{a}^{2}+W_{a}^{2}}+\sqrt{D_{b}^{2}+W_{b}^{2}}\right. \\
& \left.-\sqrt{\left(D_{a}+D_{b}\right)^{2}+\left(W_{a}+W_{b}\right)^{2}}\right\},
\end{aligned}
$$

where

$$
D_{v}=\sum_{i \in \Omega_{v}}\left(X_{i}^{2}-Y_{i}^{2}\right), \quad W_{v}=2 \sum_{i \in \Omega_{v}} X_{i} Y_{i}
$$

with $v=a, b$. The value of $\mathcal{R}_{\text {Funknown }}$ thus depends only on the statistics $D_{a}, D_{b}, W_{a}$, and $W_{b}$, which are also the parameters that completely define the profile estimators of the DOP in regions $a$ and $b$ (see [15]).

We can give an interesting interpretation of this result. Indeed, let us define the $2-\mathrm{D}$ vectors $\mathbf{a}=\left(D_{a}, W_{a}\right)^{T}$ and $\mathbf{b}$ $=\left(D_{b}, W_{b}\right)^{T}$, where ${ }^{T}$ denotes transposition. Equation (7) can be written $\mathcal{R}_{\text {Funknown }}=(\|\mathbf{a}\|+\|\mathbf{b}\|-\|\mathbf{a}+\mathbf{b}\|) / 4 \sigma^{2}$, where $\|$. is the classic norm in a Euclidean space. The triangle inequality gives $\|\mathbf{a}\|+\|\mathbf{b}\| \geqslant\|\mathbf{a}+\mathbf{b}\|$ and implies that $\mathcal{R}_{\text {Funknown }} \geqslant 0$, which is a fundamental property of a GLRT.

The expression of the GLRT is more involved than in the case where $\mathbf{F}$ is known, and it is difficult to determine the PDF of $\mathcal{R}$ in hypotheses $H_{0}$ and $H_{1}$. However, in order to determine approximately on which parameters the performance of the GLRT depends, we consider the limit of the expression of the the GLRT as the noise variance $\sigma^{2}$ tends to zero. This amounts to making the the following assumptions:

$$
X_{i}^{\sigma=0}=F_{i} m_{X},
$$

$$
Y_{i}^{\sigma=0}=F_{i} m_{Y} .
$$

If we substitute this model in the expression of the GLRT [see Eq. (7)], we obtain

$$
\mathcal{R}_{\text {Funknown }}^{\sigma=0}=\frac{S N R_{F_{a}} S N R_{F_{b}}}{4} \times Q\left[1-\sqrt{1-\left(\frac{2 \Delta P}{Q}\right)^{2}}\right],
$$

where

$$
\begin{aligned}
S N R_{F_{u}} & =\left(\sqrt{\Phi_{u}} I_{u}\right) /(\sqrt{2} \sigma), \\
\Delta P & =P_{a}-P_{b}, \\
Q & =\frac{S N R_{F_{a}}}{S N R_{F_{b}}}\left(1+P_{a}^{2}\right)+\frac{S N R_{F_{b}}}{S N R_{F_{a}}}\left(1+P_{b}^{2}\right) .
\end{aligned}
$$

This result is very interesting since it shows that if $\Delta P=0$, then $\mathcal{R}_{\text {Funknown }}^{\sigma=0}=0$, which was not obvious when considering the expression of the detector in Eq. (4). In other words, detection is not possible if the OSC contrast is zero whatever the value of the intensity contrast. This is understandable since, as shown in Eq. (C3), the fact that the $F_{i}$ are unknown makes the parameters $I_{a}$ and $I_{b}$ unidentifiable. In summary, $\mathcal{R}_{\text {Funknown }}$ is sensitive only to OSC contrast and is unable to detect intensity contrast, since the latter cannot be distinguished from the nonuniformities of the illumination.

\section{POTENTIAL GAIN WHEN USING THE GLRT ADAPTED TO NONUNIFORM ILLUMINATION}

In cases where $\mathbf{F}$ is unknown, two detection strategies are possible. The first is to use the detector $\mathcal{R}_{\text {Funknown }}$ to suppress the influence of illumination nonuniformities. However, this may not be the better strategy. Indeed, this detector does not take into account intensity contrast between the target and the background, and this information may be relevant if the illumination nonuniformities are mild. In this case, it may be preferable to apply the optimal detector for uniform illumination $\mathcal{R}_{\text {uni }}$ [see Eq. (5)]. We study this issue in Sebsection 5.B, but before, we will determine in Subsection 5.A the statistical properties of $\mathcal{R}_{\text {uni }}$ in the presence of illumination nonuniformities.

\section{A. Performance of $\mathcal{R}_{\text {uni }}$ in the Presence of Nonuniform Illumination}

Let us substitute the model of nonuniform illumination [see Eq. (1)] in the expression of the GLRT adapted to uniform illumination $\mathcal{R}_{\text {uni }}$ [see Eq. (5)]. Our objective is to determine the PDF of $\mathcal{R}_{\text {uni }}$ in hypotheses $H_{1}$ and $H_{0}$. Let us denote $\mathcal{R}_{\text {uni }}^{0}\left(\mathcal{R}_{\text {uni }}^{1}\right)$ the expression of $\mathcal{R}_{\text {uni }}$ in hypothesis $H_{0}\left(H_{1}\right)$. After some simple computations, it can be shown that $2 \mathcal{R}_{\text {uni }}^{1}$ is a noncentral chi-square random variable with two degrees of freedom and noncentrality parameter equal to

$$
\mathcal{C}^{\prime}=\left(S N R_{a}^{\prime}-S N R_{b}^{\prime}\right)^{2}+\left(S N R_{a}^{\prime} P_{a}-S N R_{b}^{\prime} P_{b}\right)^{2},
$$

where 


$$
S N R_{v}^{\prime}=\sqrt{\frac{N_{a} N_{b}}{N} \frac{\bar{F}_{v} I_{v}}{\sqrt{2} \sigma}}, \quad \bar{F}_{v}=\frac{1}{N_{v}} \sum_{i \in \Omega_{v}} F_{i}
$$

with $v=a, b$. The value of $\bar{F}_{v}$ represents the mean value of the illumination in the subsample $\Omega_{v}$. In hypothesis $H_{0}$, $2 \mathcal{R}_{\text {uni }}^{0}$ is also a noncentral chi-square random variable with two degrees of freedom and noncentrality parameter equal to

$$
\mathcal{C}^{\prime \prime}=\frac{I^{2}\left(1+P^{2}\right)}{2 \sigma^{2}} \frac{N_{a} N_{b}}{N}\left(\bar{F}_{a}-\bar{F}_{b}\right)^{2} .
$$

This results demonstrates that the detector $\mathcal{R}_{u n i}$ is not a CFAR detector, and that $\mathcal{C}^{\prime}$ or $\mathcal{C}^{\prime \prime}$ cannot be considered contrast parameters. If the sample is homogeneous (hypothesis $H_{0}$ ) but $\bar{F}_{a} \neq \bar{F}_{b}$ because of the illumination nonuniformity, the detector $\mathcal{R}_{\text {uni }}$ interprets the illumination nonuniformity as an intensity contrast and leads to a false alarm. This is not the case of the detector $\mathcal{R}_{\text {Funknown }}$, which is insensitive to intensity contrast.

\section{B. Receiver Operating Characteristics}

We have plotted on Fig. 1 and Fig. 2 the ROC of detectors $\mathcal{R}_{\text {Fknown }}, \mathcal{R}_{\text {Funknown }}$, and $\mathcal{R}_{\text {uni }}$ with three different types of nonuniform illuminations (that is, different values of $\bar{F}_{a}$ and $\bar{F}_{b}$ ). The three illumination vectors $\mathbf{F}$ are chosen so

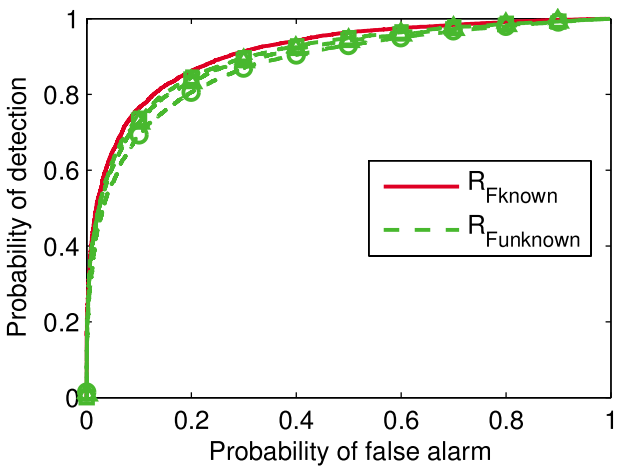

(a)

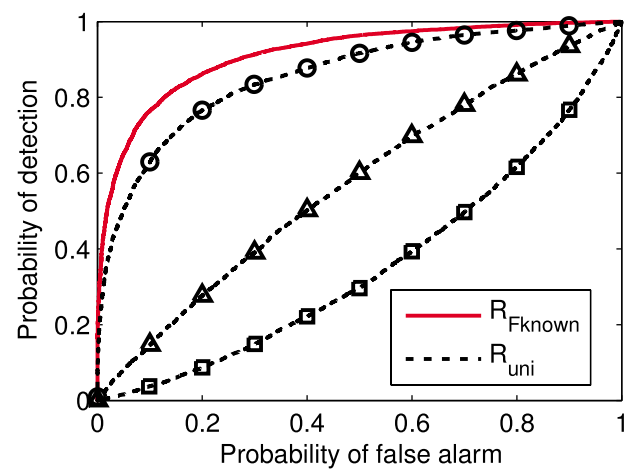

(b)

Fig. 1. (Color online) ROC curves of the detectors: (a) $\mathcal{R}_{\text {Fknown }}$ (solid), $\mathcal{R}_{\text {Funknown }}$ (dashed). (b) $\mathcal{R}_{\text {Fknown }}$ (solid) $\mathcal{R}_{\text {uni }}$ (dashed) with three different types of nonuniform illumination in scenario 1 . The contrast $\mathcal{C}_{\text {Fknown }}$ is set to 9 , and $\bar{F}_{a} / \bar{F}_{b}=1(\bigcirc), \bar{F}_{a} / \bar{F}_{b}=1.4(\triangle)$, and $\bar{F}_{a} / \bar{F}_{b}=4.7(\square)$. Other parameters are $\left(N_{a}, I_{a}, P_{a}\right)$ $=(10,100,0.8),\left(N_{b}, I_{b}, P_{b}\right)=(10,100,0.4)$, and $\sigma=15$.

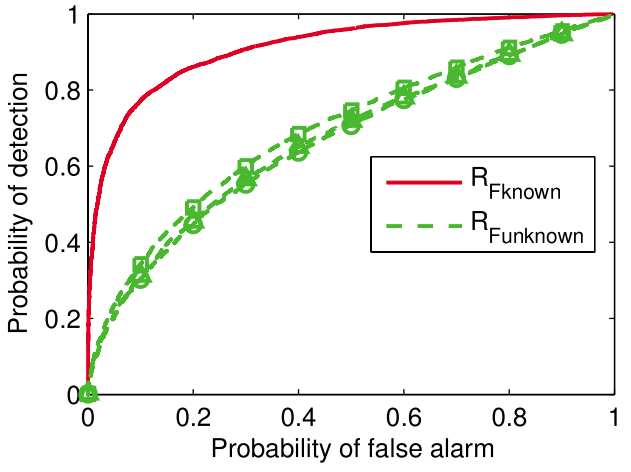

(a)

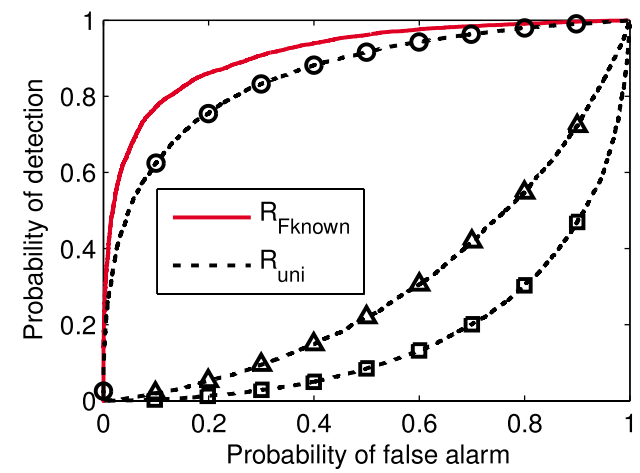

(b)

Fig. 2. (Color online) Same as in Fig. 1 in scenario 2 which corresponds to $\left(N_{a}, I_{a}, P_{a}\right)=(10,110,0.7),\left(N_{b}, I_{b}, P_{b}\right)=(10,90,0.5)$.

that successively $\bar{F}_{a} / \bar{F}_{b}=1,1.4$, and 4.7 . In all cases, the contrast $\mathcal{C}_{\text {Fknown }}$ [see Eq. (6)] remains constant by adjusting the parameters $\bar{F}_{a}$ and $\bar{F}_{b}$. The ROC of the detector $\mathcal{R}_{\text {Fknown }}$ (and as a consequence its performance) is thus identical in each different situation since it depends only on $\mathcal{C}_{\text {Fknown. }}$. These results thus set the benchmark for evaluating the performances of the other detectors.

We have considered two scenarios. Scenario 1 corresponds to $\mathcal{C}_{\text {Fknown }}=9, \quad\left[N_{a}, I_{a}, P_{a}\right]=[10,100,0.8] \quad$ and $\left[N_{b}, I_{b}, P_{b}\right]=[10,100,0.4]$; that is, there is no intensity contrast but only OSC contrast. It is seen on Fig. 1(a) that the ROC corresponding to $\mathcal{R}_{\text {Funknown }}$ are very close to the benchmark $\left(\mathcal{R}_{\text {Fknown }}\right)$, which means that with an OSC contrast only, $\mathcal{R}_{\text {Funknown }}$ is close to optimality: only a little performance is lost by estimating $\mathbf{F}$. The ROC of the detector $\mathcal{R}_{\text {uni }}$ are represented on Fig. 1(b). We note that its performance significantly depends on the illumination pattern. Indeed, this detector approaches the benchmark if $\bar{F}_{a} / \bar{F}_{b}=1$ (curve $\bigcirc$ ), but it is worse than flipping a coin if $\bar{F}_{a} / \bar{F}_{b}=4.7$; its ROC curve ( $\square$ ) is actually beneath the diagonal.

Scenario 2 corresponds to $\left[N_{a}, I_{a}, P_{a}\right]=[10,110,0.7]$ and $\left[N_{b}, I_{b}, P_{b}\right]=[10,90,0.5]$, which means that both OSC and intensity contrast are present. Since the value of $\mathcal{C}_{\text {Fknown }}$ is the same as in scenario 1 , the OSC contrast is thus lower. As a consequence, it is seen on Fig. 2(a) that the performance of $\mathcal{R}_{\text {Funknown }}$ is worse than for scenario 1, since this detector takes only the OSC contrast into account. However, one notes that the ROC obtained with the different types of illuminations are still close to each other, since the performance of $\mathcal{R}_{\text {Funknown }}$ is little affected 
by the illumination pattern. The detector $\mathcal{R}_{\text {uni }}$ still strongly depends on the illumination pattern, but provides better results than $\mathcal{R}_{\text {Funknown }}$ for $\bar{F}_{a} / \bar{F}_{b}=1$ (see curve $\bigcirc$ on Fig. 2), since it takes into account both the intensity and the OSC contrast.

\section{Application to Target Detection}

In order to illustrate the conclusion of Subsection 5.B, we have applied the detectors $\mathcal{R}_{\text {Funknown }}$ and $\mathcal{R}_{\text {uni }}$ on simulated images composed of two identical targets on a uniform background. To perform detection, we scan the scene with a mask $\mathbf{M}$ composed of two parts: $\chi_{a}$, which represents the shape of the target, and $\chi_{b}$ which is the complementary of $\chi_{a}$ in $\mathbf{M}$ (see Fig. 3). The parameters $N_{a}$ and $N_{b}$ defined previously are, in this case, the number of pixels in parts $\chi_{a}$ and $\chi_{b}$, and we will use the values $N_{a}=85$ and $N_{b}=491$ (see Fig. 3). The most computation-intensive part of $\mathcal{R}_{\text {Funknown }}$ and $\mathcal{R}_{\text {uni }}$ algorithms consists of computing moving averages in subsamples $\chi_{a}$ and $\chi_{b}$. This is equivalent to matched filtering and can be performed efficiently with fast Fourier transforms.

Let us first define the illumination model. We proceed as in [17] to obtain spatial variations with controllable correlation length. For that purpose, we model the illumination as the realization of a spatially correlated random field. We consider a Gaussian random field $g(u, v)$ with zero mean, standard deviation $\sigma_{F}$, and whose autocorrelation function is an isotropic exponential of width $l_{c}$,

$$
C^{g g}(m, n)=\sigma_{F}^{2} \exp \left(-\frac{\sqrt{m^{2}+n^{2}}}{l_{c}}\right) .
$$

We then define the illumination as $F(u, v)=[1+g(u, v)]^{2}$. The two parameters of this model are $l_{c}$, which controls the correlation length of the spatial variations, and $\sigma_{F}$, which controls the variance of the illumination pattern. In the following, we consider three values of $l_{c}, l_{c}=1,10$, and 100 (expressed in pixels). As illustrated on Fig. 4, when $l_{c}=1$ the correlation length of the spatial variations of the illumination $\mathbf{F}$ are much smaller than the target. When computing $\bar{F}_{a}$ and $\bar{F}_{b}$ with mask $\mathbf{M}$ we perform an average on the illumination and thus obtain $\bar{F}_{a} \approx \bar{F}_{b}$. When $l_{c}=10$ the correlation length of the illumination is close to the size of the target. We thus potentially observe a different average illumination in $\Omega_{a}$ and $\Omega_{b}$, and thus $\bar{F}_{a} \neq \bar{F}_{b}$. When $l_{c}=100$ the correlation lengths of the spatial variation of the illumination are much larger than the target. We thus have almost identical illumination on the whole mask $\mathbf{M}$, and $\bar{F}_{a} \approx \bar{F}_{b}$.
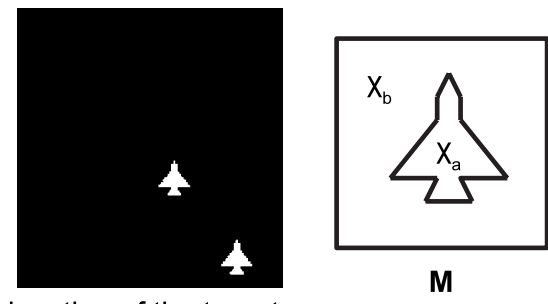

location of the targets

Fig. 3. Location of the targets on the $256 \times 256$ image, and mask $\mathbf{M}$.
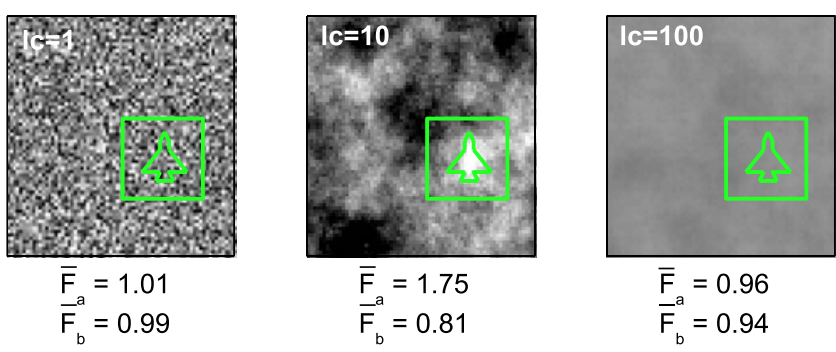

Fig. 4. (Color online) Illumination pattern $\mathbf{F}$ for $l_{c}=1,10$, and 100. The values $\bar{F}_{a}$ and $\bar{F}_{b}$ are computed for each case in the mask superimposed on the images.

We consider the same scenarios as in Fig. 1 and Fig. 2: scenario 1 corresponds to an OSC contrast only (see Fig. 5 ) and scenario 2 to both intensity and OSC contrasts (see Fig. 6), the overall contrast $\mathcal{C}_{\text {Fknown }}$ being identical in the two scenarios. The parameter values are identical to those in Fig. 1 and Fig. 2 for the targets $\left(P_{a}, I_{a}\right)$ and the background $\left(P_{b}, I_{b}\right)$. On both figures, we represent successively the illumination pattern $\mathbf{F}$, the intensity image, the OSC image, and the results of $\mathcal{R}_{\text {uni }}$ and $\mathcal{R}_{\text {Funknown }}$ detection. Each row corresponds to a different value of $l_{c}\left(l_{c}\right.$ $=1,10$, and 100).

We observe on Fig. 5 that in scenario 1, the detector $\mathcal{R}_{\text {Funknown }}$ shows good performance and is able to detect the targets under the three different illuminations. This is in agreement with the fact that all the ROC are close to the benchmark in Fig. 1(a). On the other hand, the detector $\mathcal{R}_{\text {uni }}$ is less efficient than $\mathcal{R}_{\text {Funknown }}$ for all $l_{c}$ and is significantly affected by the type of illumination. Let us linger on the case $l_{c}=10$, which corresponds to an illumination pattern whose correlation length approximately matches the size of the target. In this case, situations corresponding to $\bar{F}_{a} \neq \bar{F}_{b}$ are probable, which leads to high probability of false alarm [as is seen on curves ( $\square)$ in Fig. 1(b)]. Indeed, in this case, $\mathcal{R}_{\text {uni }}$ interprets the variations of the illumination pattern as an intensity contrast. When $l_{c}=1$ or $l_{c}=100$ the correlation length of illumination is either much smaller or much larger than the size of the targets, which implies that $\bar{F}_{a} \approx \bar{F}_{b}$ [corresponding to the curve ( $\bigcirc)$ in Fig. 1(b)]. This explains why, in these cases, $\mathcal{R}_{\text {uni }}$ has better performance than when $l_{c}=10$.

We note on Fig. 6 that in scenario 2, the performance of $\mathcal{R}_{\text {Funknown }}$ still does not depend on the type of illumination but globally decreases compared to scenario 1 . This is in agreement with the phenomenon observed on ROC: the larger the contribution of intensity contrast in the global contrast $\mathcal{C}_{\text {Fknown }}$, the smaller the contrast $\mathcal{C}_{\text {Funknown }}$. In other words, $\mathcal{R}_{\text {Fknown }}$ does not exploit the intensity contrast in the scene. Concerning the detector $\mathcal{R}_{\text {uni }}$, we observe that in the cases $l_{c}=1$ and $l_{c}=100$, its performance is close to and even better than that of $\mathcal{R}_{\text {Funknown. }}$ This is related to the fact that for these two values of $l_{c}, \mathcal{R}_{u n i}$ exploits the intensity contrast without being disturbed by the nonuniformity of the illumination. When $l_{c}=10$, the detector $\mathcal{R}_{\text {uni }}$ is significantly disturbed by the illumination pattern, which is not the case of $\mathcal{R}_{\text {Funknown }}$.

To summarize, we have demonstrated that $\mathcal{R}_{\text {uni }}$ is able to detect intensity contrast as well as OSC contrast, but is 


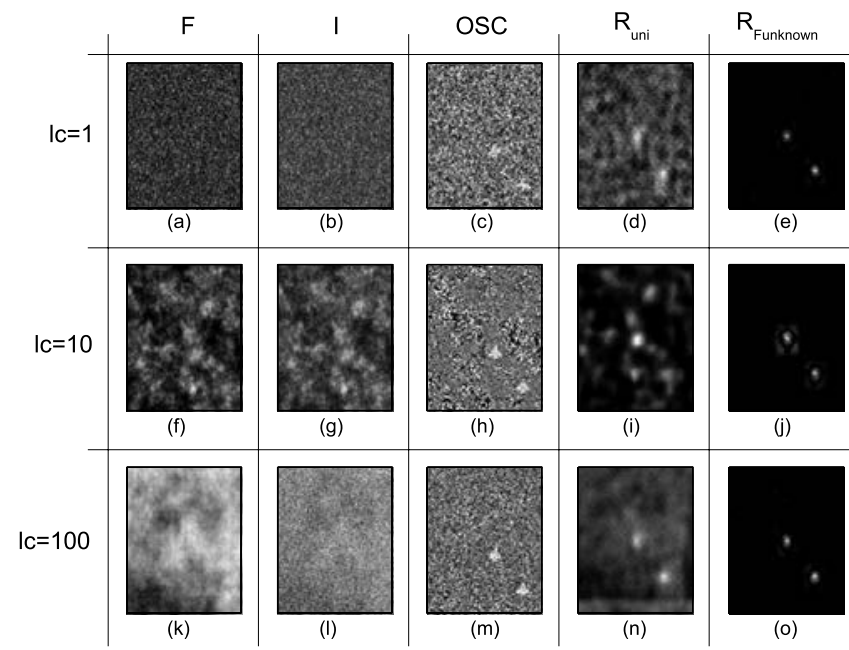

Fig. 5. Detection in scenario 1 corresponding to OSC contrast only. Horizontally: illumination pattern F, intensity image, OSCI, the results of $\mathcal{R}_{\text {uni }}$ and $\mathcal{R}_{\text {Funknown }}$ detection. Vertically: those images are computed for $l_{c}=1$, 10, and 100.

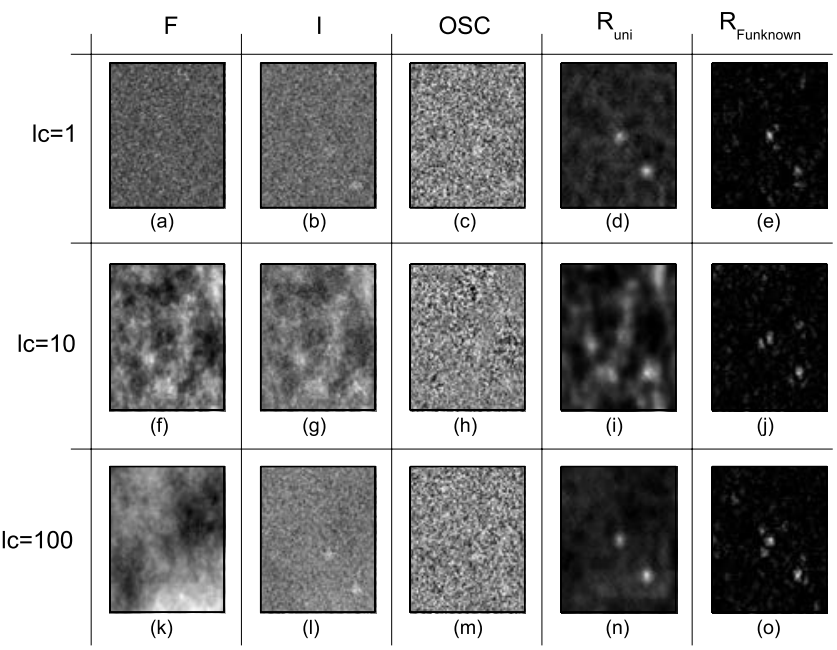

Fig. 6. Detection in scenario 2 corresponding to contrast in OSC and intensity. Horizontally: illumination pattern $\mathbf{F}$, intensity image, OSCI, the results of $\mathcal{R}_{\text {uni }}$ and $\mathcal{R}_{\text {Funknown }}$ detection. Vertically: those images are computed for $l_{c}=1,10$, and 100 .

dramatically sensitive to illumination patterns that have correlation lengths of the same order of magnitude as the target size. The detector $\mathcal{R}_{\text {Funknown }}$ is not sensitive to illumination, but does not exploit intensity contrast in the scene. The choice between the two algorithms will then depend on the nature of the contrast (OSC only or intensity and OSC), on the amplitude and correlation length of the illumination pattern, and on the target size.

\section{CONCLUSION}

We have addressed target detection in OSC images corrupted by additive detector noise and by nonuniform illumination. The expression of the GLRT was derived in different situations: uniform illumination, known nonuniform illumination, and nonuniform and unknown illumination. We have compared the detectors which assume the illumination uniform, and the one which takes into account the fact that the illumination is unknown and nonuniform. We demonstrated that the spatial distribution of the illumination and the existing contrast in the scene must be taken into account to decide between both detectors. An interesting perspective will consist of integrating in the model the photon noise and the nonlinearity of the detector, which become limits in high-flux imaging systems.

\section{APPENDIX A: DERIVATION OF THE GLRT WHEN THE ILLUMINATION IS KNOWN}

We consider that $\mathbf{F}$ is known, and that $m_{X}$ and $m_{Y}$ are unknown and thus constitute nuisance parameters. To deal with these nuisance parameters, we will use the profile likelihood method. It consists of considering them as deterministic unknowns and maximizing the log-likelihood with respect to them,

$$
\ell(\chi \mid \mathbf{F})=\underset{m_{X}, m_{Y}}{\arg \max }\left[\mathcal{L}\left(\chi \mid \mathbf{F}, m_{X}, m_{Y}\right)\right] .
$$

The obtained function $\ell(\chi \mid \mathbf{F})$ is called profile loglikelihood (PL) since it does not correspond to a real loglikelihood (its integral with respect to $\chi$ may not be equal 
to 1). Let us start with the expression of the loglikelihood,

$$
\begin{aligned}
\ell\left(\chi \mid \mathbf{F}, m_{X}, m_{Y}\right)= & -2 N \ln [\sqrt{2 \pi}]-\frac{1}{2 \sigma^{2}} \sum_{i=1}^{N}\left(X_{i}-F_{i} m_{X}\right)^{2} \\
& -\frac{1}{2 \sigma^{2}} \sum_{i=1}^{N}\left(Y_{i}-F_{i} m_{Y}\right)^{2}
\end{aligned}
$$

It has been shown in [15] that the values of $m_{X}$ and $m_{Y}$ that maximize this function are $\hat{m}_{U}=\sum_{i=1}^{N} F_{i} U_{i} / \sum_{i=1}^{N} F_{i}^{2}$ with $U=\{X, Y\}$. Let us substitute these values in the loglikelihood. One obtains

$$
\begin{aligned}
\ell(\chi \mid \mathbf{F})= & -2 N \ln [\sqrt{2 \pi}]-\frac{1}{2 \sigma^{2}}\left\{\sum_{i=1}^{N} X_{i}^{2}-\frac{\left(\sum_{i=1}^{N} F_{i} X_{i}\right)^{2}}{\sum_{i=1}^{N} F_{i}^{2}}\right\} \\
& -\frac{1}{2 \sigma^{2}}\left\{\sum_{i=1}^{N} Y_{i}^{2}-\frac{\left(\sum_{i=1}^{N} F_{i} Y_{i}\right)^{2}}{\sum_{i=1}^{N} F_{i}^{2}}\right\} .
\end{aligned}
$$

We now proceed to the determination of the GLRT. Its logarithm is defined as $\mathcal{R}_{\text {Fknown }}=\ell_{1}(\chi \mid \mathbf{F})-\ell_{0}(\chi \mid \mathbf{F})$ where $\ell_{1}(\chi \mid \mathbf{F})$ is the PL in hypothesis $H_{1}$ and $\ell_{0}(\chi \mid \mathbf{F})$ the PL in hypothesis $H_{0}$.

In hypothesis $H_{0}$, the sample is homogeneous. The PL is thus equal to that of a homogeneous region, $\ell_{0}(\chi \mid \mathbf{F})$ $=\ell(\chi \mid \mathbf{F})$. On the other hand, in hypothesis $H_{1}$, the sample consists of two independent subsamples with different parameters. The $\mathrm{PL}$ is thus the sum $\ell_{1}(\chi \mid \mathbf{F})=\ell\left(\chi_{a} \mid \mathbf{F}\right)$ $+\ell\left(\chi_{b} \mid \mathbf{F}\right)$. Substituting these expressions of $\ell_{0}(\chi \mid \mathbf{F})$ and $\ell_{1}(\chi \mid \mathbf{F})$ in the definition of the GLRT, we obtain Eq. (4).

\section{APPENDIX B: DERIVATION OF $\mathcal{R}_{\text {Fknown }}^{0}$ AND $\mathcal{R}_{\text {Fknown }}^{1}$}

The detection performance depends on the statistical parameters of the GLRT in hypotheses $H_{1}$ and $H_{0}$. Let us denote $\mathcal{R}_{\text {Fknown }}^{0}\left(\mathcal{R}_{\text {Fknown }}^{1}\right)$ the expression of the GLRT $\mathcal{R}_{\text {Fknown }}$ in hypothesis $H_{0}\left(H_{1}\right)$. Let us study these two random variables.

In hypothesis $H_{0}$, one has $m_{U}^{a}=m_{U}^{b}=m_{U}^{0}$ where $U$ $=X, Y$. Consequently,

$$
\begin{aligned}
& \hat{m}_{U}^{a}=m_{U}^{0}+n_{U}^{a} / \Phi_{a}, \\
& \hat{m}_{U}^{b}=m_{U}^{0}+n_{U}^{b} / / \Phi_{b},
\end{aligned}
$$

where $n_{U}^{v}=\Sigma_{i \in \Omega_{v}} F_{i}\left[n_{U}\right]_{i}$ with $U=\{X, Y\}$, and $v=\{a, b\}$ represent independent Gaussian noise with zero average and variance equal, respectively, to $\sigma^{2} \Phi_{a}$ and $\sigma^{2} \Phi_{b}$. Consequently, the random value $\mathcal{R}_{\text {Fknown }}^{0}$ has the form

$$
\mathcal{R}_{\text {Fknown }}^{0}=\frac{\left(\Phi_{b} n_{a}^{X}-\Phi_{a} n_{b}^{X}\right)^{2}+\left(\Phi_{b} n_{a}^{Y}-\Phi_{a} n_{b}^{Y}\right)^{2}}{2 \sigma^{2} \Phi_{a} \Phi_{b}\left(\Phi_{a}+\Phi_{b}\right)}=\frac{b_{X}^{2}}{2}+\frac{b_{Y}^{2}}{2},
$$

where

$$
b_{U}=\frac{\Phi_{b} n_{a}^{U}-\Phi_{a} n_{b}^{U}}{\sigma \sqrt{\Phi_{a} \Phi_{b}\left(\Phi_{a}+\Phi_{b}\right)}}
$$

for $U=X, Y$ represent independent Gaussian noise with zero mean and unit variance. Thus $2 \mathcal{R}_{\text {Fknown }}^{0}$ is a chi- square random variable with two degrees of freedom [22].

In hypothesis $H_{1}$, one has $m_{U}^{a} \neq m_{U}^{b}$, and thus

$$
\begin{aligned}
& \hat{m}_{U}^{a}=m_{a}+n_{U}^{a} / \Phi_{a}, \\
& \hat{m}_{U}^{b}=m_{b}+n_{U}^{b} / \Phi_{b},
\end{aligned}
$$

where $n_{X}^{a}$ and $n_{X}^{b}$ are defined above. After some computations one obtains the GLRT in the form

$$
\mathcal{R}_{\text {Fknown }}^{1}=\frac{1}{2}\left(\mathcal{C}_{X}+b_{X}\right)^{2}+\frac{1}{2}\left(\mathcal{C}_{Y}+b_{Y}\right)^{2}
$$

with

$$
\mathcal{C}_{U}=\sqrt{\frac{\Phi_{a} \Phi_{b}}{\left(\Phi_{a}+\Phi_{b}\right)}} \frac{\left|m_{U}^{a}-m_{U}^{b}\right|}{\sigma}
$$

and $U=X, Y$. Thus $2 \mathcal{R}_{F k n o w n}^{1}$ is a noncentral chi-square random variable with two degrees of freedom and noncentrality parameter equal to

$$
\begin{aligned}
\mathcal{C}_{\text {Fknown }} & =\mathcal{C}_{X}^{2}+\mathcal{C}_{Y}^{2} \\
& =\frac{1}{\sigma^{2}} \frac{\Phi_{a} \Phi_{b}}{\left(\Phi_{a}+\Phi_{b}\right)} \times\left[\left(m_{X}^{a}-m_{X}^{b}\right)^{2}+\left(m_{Y}^{a}-m_{Y}^{b}\right)^{2}\right] .
\end{aligned}
$$

This parameter can also be expressed as a function of the parameters $P$ and $S N R$. Indeed, by substituting Eq. (3) in Eqs. (B1) and (B2), one obtains Eq. (6).

\section{APPENDIX C: DERIVATION OF THE PROFILE LIKELIHOOD WITH UNKNOWN ILLUMINATION}

We compute the PL with unknown illumination, which consists of determining

$$
\ell(\chi)=\arg \max _{m_{X}, m_{Y}, \mathbf{F}}\left[\ell\left(\chi \mid \mathbf{F}, m_{X}, m_{Y}\right)\right] .
$$

We detail in the following how, successively, F, $m_{X}$, and $m_{Y}$ are estimated in the ML sense, and substituted in the likelihood.

\section{Elimination of $\mathbf{F}$}

Let us start with the expression of the log-likelihood in Eq. (A1). Annulling the derivative of $\ell\left(\chi \mid \mathbf{F}, m_{X}, m_{Y}\right)$ with respect to $F_{i}$ leads to

$$
\hat{F}_{i}=\frac{m_{X} X_{i}+m_{Y} Y_{i}}{m_{X}^{2}+m_{Y}^{2}} .
$$

Substituting this value into the expression of the loglikelihood yields

$$
\ell\left(\chi \mid m_{X}, m_{Y}\right)=-\frac{1}{2 \sigma^{2}} \sum_{i=1}^{N} \frac{\left(m_{y} X_{i}-m_{X} Y_{i}\right)^{2}}{m_{X}^{2}+m_{Y}^{2}},
$$

which can be expressed as a function of $\rho=m_{X} / m_{Y}$ as 


$$
\ell(\chi \mid \rho)=-\frac{1}{2 \sigma^{2}} \frac{T_{X}-W \rho+T_{Y} \rho^{2}}{1+\rho^{2}},
$$

with $T_{U}=\Sigma_{i=1}^{N} U_{i}^{2}, U=\{X, Y\}$, and $W=2 \Sigma_{i=1}^{N} X_{i} Y_{i}$. It is seen that the PL depends only on $\rho$, that is, on $P$ since $P$ $=(\rho-1) /(\rho+1)$. It does not depend on $I=m_{X}+m_{Y}$. This means than $I$ cannot be estimated from the data.

\section{Elimination of $\rho$}

In order to estimate $\rho$ in the ML sense, one determines the derivative of $\ell(\chi \mid \rho)$ with respect to $\rho$,

$$
\frac{\partial \ell}{\partial \rho}=-\frac{1}{\sigma^{2}} \frac{W \rho^{2}-2 D \rho-W}{2\left(1+\rho^{2}\right)^{2}},
$$

where $D=T_{X}-T_{Y}$. Annulling this derivative corresponds to solving the following second-order polynomial equation:

$$
W \rho^{2}-2 D \rho-W=0
$$

There are two possible solutions:

$$
\rho=\frac{D \pm \sqrt{\Delta}}{W},
$$

with

$$
\Delta=D^{2}+W^{2} .
$$

The correct solution must correspond to a maximum of the PL. For that purpose, let us compute the second derivative of $\ell(\chi \mid \rho)$ with respect to $\rho$ :

$$
\frac{\partial^{2} \ell}{\partial \rho^{2}}=-\frac{1}{2 \sigma^{2}} \frac{-2 W \rho^{3}+6 D \rho^{2}+6 W \rho-2 D}{\left(1+\rho^{2}\right)^{4}} .
$$

We are interested in the value of this second derivative at the points $\rho^{*}$ where the derivative is zero. As seen above, these points verify the relation (C5). Substituting this relation into Eq. (C8), one obtains

$$
\frac{\partial^{2} \ell}{\partial \rho^{2}}=-\frac{2}{\sigma^{2}} \frac{\rho / W\left(D^{2}+W^{2}\right)}{\left(1+\rho^{2}\right)^{4}} .
$$

To show a maximum, the second derivative must be negative, which implies $\rho / W \geqslant 0$. Using the expression of $\rho$ in Eq. (C6), the condition becomes

$$
\frac{D \pm \sqrt{D^{2}+W^{2}}}{W^{2}} \geqslant 0 .
$$

The only solution which corresponds to a maximum of the $\mathrm{PL}$ is thus

$$
\hat{\rho}=\frac{D+\sqrt{D^{2}+W^{2}}}{W} .
$$

One can note that this solution can also be written as

$$
\hat{\rho}=r+\operatorname{sgn}(W) \sqrt{1+r^{2}},
$$

with $r=D / W$ and $\operatorname{sgn}(x)=1$ if $x>0$ and -1 otherwise.

\section{Profile Log-Likelihood}

This estimate of $\rho$ is substituted in the expression of $\ell(\chi \mid \rho)$ [see Eq. (C3)] to find the final PL,

$$
\ell(\chi \mid \hat{\rho})=-\frac{1}{4 \sigma^{2}}\left(2 T_{Y}-\frac{\sqrt{\Delta}}{1+\frac{D^{2}}{W^{2}}+\frac{D \sqrt{\Delta}}{W^{2}}}\right),
$$

where we have taken into account that $D=T_{X}-T_{Y}$. Let us consider the term

$$
\frac{\sqrt{\Delta}}{1+\frac{D^{2}}{W^{2}}+\frac{D \sqrt{\Delta}}{W^{2}}}=\frac{W^{2} \sqrt{\Delta}}{W^{2}+D^{2}+D \sqrt{\Delta}} .
$$

Taking into account that by definition $\Delta=D^{2}+W^{2}$, one has

$$
\frac{W^{2} \sqrt{\Delta}}{W^{2}+D^{2}+D \sqrt{\Delta}}=\sqrt{\Delta}-D .
$$

Consequently,

$$
\ell(\chi \mid \hat{\rho})=-\frac{1}{4 \sigma^{2}}\left(T_{X}+T_{Y}-\sqrt{\left(T_{X}-T_{Y}\right)^{2}+W^{2}}\right) .
$$

Replacing $T_{X}, T_{Y}$, and $\Delta$ with their expressions as a function of the data $X_{i}$ and $Y_{i}$, one obtains

$$
\begin{aligned}
\ell(\chi \mid \hat{\rho})= & -\frac{1}{4 \sigma^{2}}\left\{\sum_{i=1}^{N}\left(X_{i}^{2}+Y_{i}^{2}\right)\right. \\
& \left.-\sqrt{\left(\sum_{i=1}^{N}\left(X_{i}^{2}-Y_{i}^{2}\right)\right)^{2}+4\left(\sum_{i=1}^{N} X_{i} Y_{i}\right)^{2}}\right\} .
\end{aligned}
$$

This is the expression of the PL of a homogeneous region.

\section{APPENDIX D: DERIVATION OF THE GLRT WHEN THE ILLUMINATION IS UNKNOWN}

We determine the expression of the GLRT when the illumination is unknown. We use the PL of a homogeneous region obtained in Eq. (C12) and compute its expression in hypotheses $H_{0}$ and $H_{1}$. In hypothesis $H_{0}$, the sample is considered homogeneous. Consequently, its PL is

$$
\ell_{0}(\chi)=-\frac{1}{4 \sigma^{2}}\left\{\left(T_{X}+T_{Y}\right)-\sqrt{\left(T_{X}-T_{Y}\right)^{2}+W^{2}}\right\},
$$

with $T_{U}=\Sigma_{i=1}^{N} U_{i}^{2}, U=\{X, Y\}$, and $W=2 \sum_{i=1}^{N} X_{i} Y_{i}$. Those parameters are computed on the whole sample $\chi$.

In hypothesis $H_{1}$, the sample is divided into two parts, assumed to have different parameters. Consequently, its generalized log-likelihood is

$$
\begin{aligned}
\ell_{1}(\chi)= & \ell\left(\chi_{a}\right)+\ell\left(\chi_{b}\right)=-\frac{1}{4 \sigma^{2}}\left\{\left(T_{X}+T_{Y}\right)-\sqrt{\left(T_{X}^{a}-T_{Y}^{a}\right)^{2}+W_{a}^{2}}\right. \\
& \left.-\sqrt{\left(T_{X}^{b}-T_{Y}^{b}\right)^{2}+W_{b}^{2}}\right\}
\end{aligned}
$$

where $T_{U}^{v}=\Sigma_{i \in \Omega_{v}} U_{i}^{2}$ and $W^{v}=2 \Sigma_{i \in \Omega_{v}} X_{i} Y_{i}$, with $U=\{X, Y\}$ 
and $v=\{a, b\}$. Indeed, one notes that $T_{X}=T_{X}^{a}+T_{X}^{b}, T_{Y}=T_{Y}^{a}$ $+T_{Y}^{b}$, and $W=W_{a}+W_{b}$. The expression of the generalized log-likelihood ratio, defined as $\mathcal{R}_{\text {Funknown }}=\ell_{1}(\chi)-\ell_{0}(\chi)$, is given in Eq. (7).

\section{ACKNOWLEDGMENTS}

Arnaud Bénière's Ph.D. thesis is supported by the Délégation Générale pour l'Armement (DGA), MRIS domain IMAT (contact Jacques Blanc-Talon).

\section{REFERENCES}

1. L. B. Wolff, "Polarization vision: a new sensory approach to image understanding," Image Vis. Comput. 15, 81-93 (1997).

2. P. J. Wu, J. Joseph, and T. Walsh, "Stokes polarimetry imaging of rat tail tissue in a turbid medium: degree of linear polarization image maps using incident linearly polarized light," J. Biomed. Opt. 11, 014031 (2006).

3. J. M. Bueno, J. Hunter, C. Cookson, M. Kisilak, and M. Campbell, "Improved scanning laser fundus imaging using polarimetry," J. Opt. Soc. Am. A 24, 1337-1348 (2007).

4. M. Alouini, F. Goudail, A. Grisard, J. Bourderionnet, D. Dolfi, I. Baarstad, T. Løke, P. Kaspersen, and X. Normandin, "Active polarimetric and multispectral laboratory demonstrator: contrast enhancement for target detection," Proc. SPIE 6396, 63960B (2006)

5. P. Terrier, V. Devlaminck, and J. M. Charbois, "Segmentation of rough surfaces using a polarization imaging system," J. Opt. Soc. Am. A 25, 423-430 (2008).

6. S. Breugnot and P. Clémenceau, "Modeling and performances of a polarization active imager at $\lambda=806 \mathrm{~nm}$," Opt. Eng. 39, 2681-2688 (2000).

7. R. E. Nothdurft and G. Yao, "Effects of turbid media optical properties on object visibility in subsurface polarization imaging," Appl. Opt. 45, 5532-5541 (2006).

8. M. Anastasiadou, A. De Martino, D. Clement, F. Lige, B. Laude-Boulesteix, N. Quang, J. Dreyfuss, B. Huynh, A. Nazac, L. Schwartz, and H. Cohen, "Polarimetric imaging for the diagnosis of cervical cancer," Phys. Status Solidi C 5, 991-999 (2008).
9. P. Terrier and V. DeVlaminck, "Robust and accurate estimate of the orientation of partially polarized light from a camera sensor," Appl. Opt. 40, 5233-5239 (2001).

10. J. C. Ramella-Roman, K. Lee, S. A. Prahl, and S. L. Jacques, "Polarized light imaging with a handheld camera," Proc. SPIE 5068, 284-293 (2003).

11. F. Goudail and P. Réfrégier, "Statistical techniques for target detection in polarization diversity images," Opt. Lett. 26, 644-646 (2001).

12. M. Roche, J. Fade, and P. Réfrégier, "Parametric estimation of the square degree of polarization from two intensity images degraded by fully developed speckle noise," J. Opt. Soc. Am. A 24, 2719-2727 (2007).

13. P. Réfrégier, M. Roche, and F. Goudail, "Cramer-Rao lower bound for the estimation of the degree of polarization in active coherent imagery at low photon level," Opt. Lett. 31, 3565-3567 (2006).

14. V. L. Gamiz and J. F. Belsher, "Performance limitations of a four-channel polarimeter in the presence of detection noise," Opt. Eng. 41, 973-980 (2002).

15. A. Bénière, F. Goudail, M. Alouini, and D. Dolfi, "Degree of polarization estimation in the presence of nonuniform illumination and additive Gaussian noise," J. Opt. Soc. Am. A 25, 919-929 (2008).

16. A. Bénière, F. Goudail, M. Alouini, and D. Dolfi, "Estimation precision of degree of polarization in the presence of signal-dependent and additive Poisson noises," J. Eur. Opt. Soc. Rapid Publ. 3, 08,002 (2008).

17. F. Goudail and P. Réfrégier, "Target segmentation in active polarimetric images by use of statistical active contours," Appl. Opt. 41, 874-883 (2002)

18. O. Germain and P. Réfrégier, "Snake-based method for the segmentation of objects in multichannel images degraded by speckle," Opt. Lett. 24, 814-816 (1999).

19. M. Alouini, F. Goudail, N. Roux, L. Le Hors, P. Hartemann, S. Breugnot, and D. Dolfi, "Active spectro-polarimetric imaging: signature modeling, imaging demonstrator and target detection," Eur. Phys. J.: Appl. Phys. 42, 129-139 (2008).

20. S. M. Kay, Fundamentals of Statistical Signal Processing, Vol. I, Estimation Theory (Prentice-Hall, 1993).

21. A. Papoulis, Probability, Random Variables and Stochastic Processes (McGraw-Hill, 1991).

22. E. T. Jaynes, Probability Theory: The Logic of Science (Cambridge U. Press, 1995). 